www.jmscr.igmpublication.org

Impact Factor 5.84

Index Copernicus Value: 83.27

ISSN (e)-2347-176x ISSN (p) 2455-0450

crossref DOI: https://dx.doi.org/10.18535/jmscr/v5i2.18

Journal Of Medical Science And Clinical Research

IGM Publication

An Official Publication of IGM Publication

\title{
Cerebral Encephalitis- A Rare CNS Complication of Scorpion Sting
}

\author{
Authors \\ Dr Babita Ghodke ${ }^{1}$, Dr Atul S. Rai ${ }^{2}$, \\ ${ }^{1}$ Associate Professor, ${ }^{2}$ Third Year Resident \\ Department of General Medicine, MGM Medical College, MGM Institute of Health Sciences \\ [Deemed University, U/S.3 of UGC Act 1956] Navi Mumbai INDIA
}

\begin{abstract}
Objectives- Scorpion envenomation is an important public health hazard in tropical and subtropical region. Envenomation by scorpion can result in a wide range of clinical effects including cardiotoxicity, respiratory dysfunction and a rare but important complication affecting the central nervous system being stroke both ischaemic and haemorrhagic.

There are about 1500 scorpion species known to exist, of which 30 are of medical importance. In India, the Indian Red Scorpion (Mesobuthus tamulus) common in Western Maharashtra, Gujarat, Kerala, Andhra Pradesh, Tamil Nadu and Karnataka, is known to cause focal neurological presentations which include hemiparesis, haemorrhagic or thrombotic stroke with poor outcome.

Methods

Case Report- A 45-year-old male, non hypertensive, non diabetic from a rural area (Raigad district), was brought to MGM Medical College and Hospital by relatives with a history of scorpion sting on the proximal phalanx of second toe of the left foot.

The patient presented in the emergency room with throbbing pain at the site of sting, profuse sweating, and rapid breathing with Glasgow coma scale of 12/15. His vitals were unstable with a blood pressure of 180/100 $\mathrm{mmHg}$ and a heart rate of 56 beats/min. Complete haemogram, renal and liver function tests, serum electrolytes were within normal limits. CPK MB levels were not raised. Chest radiograph showed evidence of bilateral pulmonary edema. Patient was admitted in the intensive care unit and was managed as a critical case. After 3 days of admission, patient developed unexplained unconsciousness. CT brain showed no evidence of intracranial bleed and MRI brain was suggestive of focal encephalitis in frontal lobe. CSF examination was done which was within normal limits, thus indicating the probable cause of encephalitis to be scorpion envenomation.

Patient was treated with prazosin and other supportive measures. However, there was no clinical improvement and the patient expired.
\end{abstract}

\section{Introduction}

Scorpion envenomation is an important public health hazard in tropical and sub-tropical region. scorpion envenomation is an occupational hazard for farmers, farm labors, villagers, migrating population and hunters. Scorpions are generally found in dry, hot environments There are about 1500scorpion species known to exist about 30 are of medical importance

In India Indian RED SCORPIO [MESOBUTHUS TAMULUS] known to cause focal neurological presentation include hemiparesis, hemorrhagic or 
thrombotic ischemic stroke with poor outcome. Envenomation by scorpion can result in a wide range of clinical effects ranging from local manifestations to systemic complications.

\section{Epidemiology}

The annual number of scorpion stings cases exceeds 1.23 million of which more of cases encounter fatal complications. Real incidence, morbidity \& deaths are scarce, because most of victims don't seek medical treatment \& prefer to consult traditional healers.

In Asia epidemiological data on scorpion stings scarce. INDIA is the most affected with a reported incidence of $0.6 \% .1$ mostly during hot months MARCH to JUNE and SEPTEMBER to OCTOBER at endemic areas i.e. western Maharashtra, Karnataka, Andhra Pradesh, Saurashtra, and Tamil Nadu.

\section{Scorpion Vaenom}

Venom is deposited in skin deep to subcutaneous tissue, almost complete absorption of the venom from sting site would occur in 7-8 hours. $70 \%$ of maximum concentration of venom in the blood reached within 15 minutes and time needed to reach maximum venom blood concentration is $101 \pm 8$ minutes in experimental animals, half life of intravenously injected venom is between 4 to 7 minutes and takes 4.2 to 13.4 hours for elimination from blood ${ }^{2,8,9,11}$.

Several low molecular weight basic proteins, neurotoxins, nucleotides, aminoacids, oligopeptides, cardipotoxins, nephrotoxin, hemolytic toxins, phosphodiesterase, phospholipase A, hyaluroinidase. Acetylcholineesterase, glycosaminoglycans, histamine, serotonin. 5-hydroxyptamine and proteins that inhibit protease, angiotnsinase and succinate -dehydrogenese, ribonuclease, 5- nucleotidase. Multiple toxins may be present in the venom of a single species of scorpion capable to produce a potent synergic effects in victim. ${ }^{3,4}$ Neurotoxins of scorpion venom content is highly lethal than neurotoxin of snake venom .
The yellow scorpion Leiurus Quinquestriatus (LQ) and Mesobuthus Tamulushave been reported among the most lethal scorpion species.The main molecular targets of scorpion neurotoxins are the voltage gated sodium channels and potassium channels including calcium activated potassium channels, explained scorpion neurotoxins act mainly on excitable cells of nerves and muscles

Iberiotoxin and tamulotoxin content of scorpion Mesobuthus Tamulusvenom are the only selective inhibitor of potassium channel and blocking effects of scorpion toxins on the potassium channel the action potential across excitable cell membrane becomes prolonged.

\section{Clinical Manifestations}

Local manifestations severe excruciating radiating pain from sting site. Edema and inflammation at site of sting.Systemic manifestations vomiting due to autonomic storm mostly seen with sting of mesobuthus tamulus. profuse sweatin, salivation [thick ropy salivation], priapism, mydriasis, cardiovascular [ hypertension with bradycardia in 45 to $70 \%$ cases, tachycardia in 15 to $20 \%$ cases]. 888 scorpion sting cases studied during 19 years at primary heath centers over western coast of Maharasahtra of theses 167 (19\%) had pulmonary $5,16,17$ edema.

Mesobuthus Tamulusmay cause focal neurological presentation include hemi paresis, hemorrhagic or thrombotic stroke. DIC is main contributory factors for neurological manifestations.

Case Report [From MGM Medical College And Hospital, Kamothe, Sector-18 Navi Mumbai, Maharashtra]

A 45 years old male from rural area RAIGAD district non diabetic non hypertensive brought by relatives to MGM MEDICAL COLLEGE AND HOSPITAL with alleged history of scorpion sting over proximal phalanx of second toe of the left foot.The patient presented in the emergency room with throbbing pain at the site of sting, profuse sweating, priapism, and rapid breathing. 
Examination reveals GCS [GLASGOW COMA SCALE] was $12 / 15$ with no neurological evident manifestations. Heart Rate 56/minute,Blood pressure 180/100 mm hg. Respiratory rate 34/minute. Systemic examination: cardio vascular, respiratory, per abdominal, central nervous system were within normal limits. Patient was admitted under critical care unit where after 3 days of admission patient developed unexplained unconsciousness.

\section{Investigations Do}

BLOOD investigations: Complete haemogram with in normal limits, liver and renal function were within normal limits, serum electrolytes with in normal limits. CPK MB levels not raised.

REDIOLOGICAL investigations:

CHEST X-RAY shows unilateral diffuse oedema CT-BRAIN and MRI BRAIN imaging done showing Focal FLAIR hyperintensity in sub cortical region of right frontal lobe suggestive of ENCEPHALITIS.

CSF examination NORMAL no abnormality seen. Treatment given: in form of supportive care, tab. prazosine $2.5 \mathrm{mg}$, tab. alprazolam $0.25 \mathrm{mg}$, inj. tetanus toxide $0.5 \mathrm{ml}$ deep I.M. inj. Avil 1 ampule I.V.Inspite of giving all supportive care patient died on $5^{\text {th }}$ day of hospitalization.

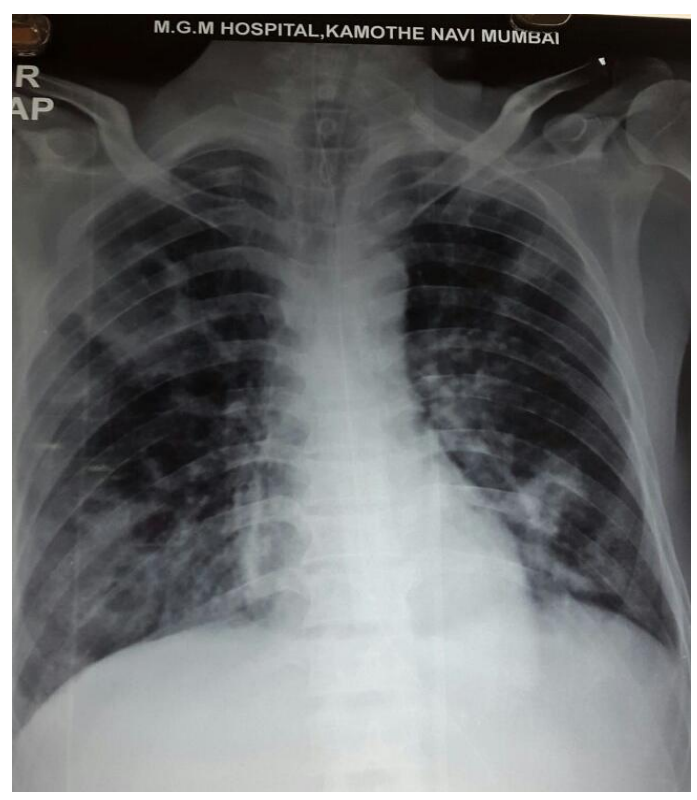

Fig. 1 CHEST X-RAY showing bilaterally diffuse pulmonary edema

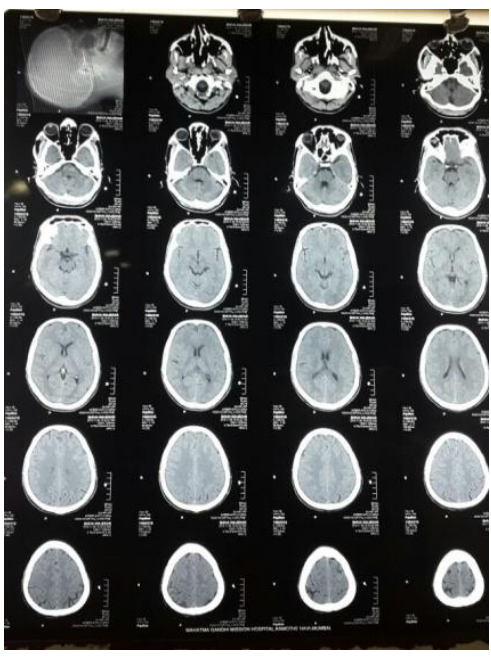

Figure 2 CT Brain

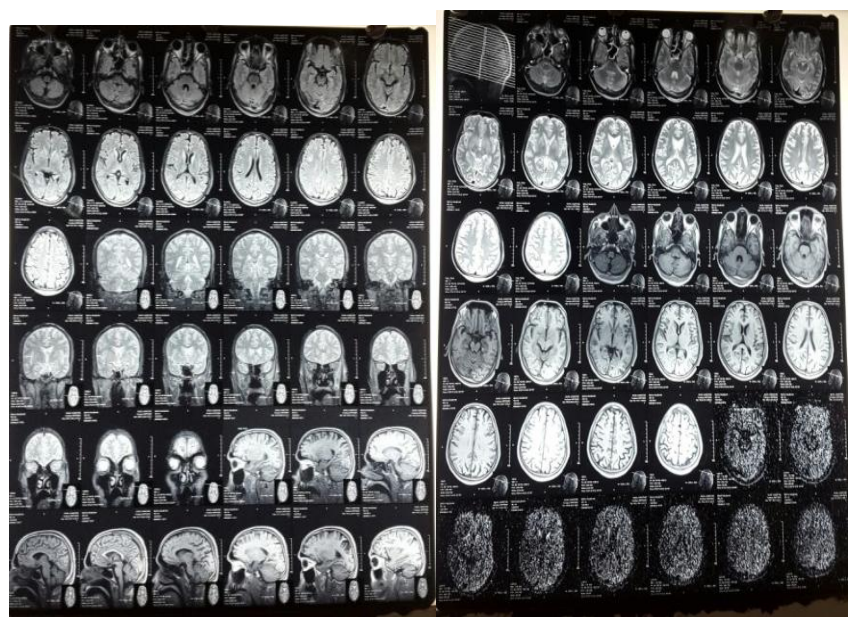

Figure 3 And 4 MRI- Brain

\section{CONCLUSION}

Neurological complications of scorpion envenomation is not only limited to Hemorrhagic or thrombotic stroke sometimes it shows variability inform of encephalitis.

CNS complications contribute to about $2 \%$ of all other complications due to scorpion envenomation. Despite adequate treatment and supportive measures, involvement of CNS in cases of scorpion envenomation carries a very bad prognosis. Proper medical management may reduce the risk of neurological complications which in turn reduce the mortality and improve the functional outcome of survivors.

\section{REFERENCE}

1. Chippaux JP and Goyffon M. Epidemiology of scorpionism: a global appraisal. Acta Trop 2008;107:71-9. 
2. Ismail $\mathrm{M}$ and Abd-elsalam MA. Are the toxicological effects of scorpion envenomation related to tissue venom concentration? Toxicon 1988;233-56.

3. Possani LD, Baltazar B, Delepierre M and Tygat J. Scorpion toxins specific for NA+ channels. Eur J Biochem 1999;264:287300 .

4. Gwee MCE, Nirtthanan S, Khoo H , Gopalkrishnakone P, Kini MR, Cheah LS. Autonomic effects of same scorpion venoms and toxins. Clinical experimental pharmacology and Physiology 2002;29: 795-801.

5. Bawaskar HS and Bawaskar PH. Envenoming by scorpions and snakes (elapidae) their neurotoxins and Therapeutics. Trop Doct 2000;30:23-25.

6. Sarakar S, Bhattacharya P and Paswan A. Cerebrovascular manifestations and alteration of coagulation profile in scorpion sting; a case series. Indian J Crit Care 2008;12:15-17.

7. Possani LD, Baltazar B, Delepierre M and Tygat J. Scorpion toxins specific for NA+ channels. Eur J Biochem 1999;264:287300 .

8. Strong Pn, Clark GS, Aamugan A, DeAllie FA et al. Tamulotoxin : a novel potassium channel blocker from venom of the Indian red scorpion Mesobuthus Tamulus. Arch Biochem Biophy 2001;385:138-44.

9. Gwee MCE, Nirtthanan S, Khoo H , Gopalkrishnakone P, Kini MR, Cheah LS. Autonomic effects of same scorpion venoms and toxins. Clinical experimental pharmacology and Physiology 2002;29: 795-801.

10. Gueron $\mathrm{M}$, and yaron r. Cardiovascular manifestations of severe scorpion sting. Chest 1970;57:156-62.
11. Bawaskar HS. Diagnostic cardiac Premonitory signs and symptoms of red scorpion sting. Lancet 1982;2:552-54.

12. Bawaskar HS and Bawaskar PH. ' Sting by red scorpion (Buthotus Tamulus) in Maharashtra state,India: a clinical study. Trans Roy Soc Trop Med Hyg 1989;83:858-60.

13. Ismail $M$ and Abd-elsalam MA. Are the toxicological effects of scorpion envenomation related to tissue venom concentration? Toxicon 1988;233-56.

14. Ismail M. The scorpion envenoming syndrome. Toxicon 1995;33:825- 58

15. Corrodo AP, neto Fr and Antonio A. The mechanism of the hypertensive effects of Brazilian scorpion venom (Tityus Serrulatus Lutz e Mellao). Toxicon 1974;12:145-50.

16. La Grange rG. Elevation of blood pressure and plasma rennin level by venom from scorpion,Centruoides sculpturatus and Leiurus Quinquestriatus. Toxicon 1977;15:429-33.

17. Murthy KEK and Vakil AE. Elevated of plasma angiotensin levels in dogs by Indian red scorpion (Buthus Tamulus) venom and its reversal by administration of insulin + tolazolin. Indian J Med Res 1988;88:376-79. 